\title{
How much access to laboratories should veterinarians have?
}

\author{
Jerald Silverman, DVM
}

Many researchers are understandably reluctant to have people wandering through their laboratory. Not only is it an interruption of the ongoing work, but it can lead to errors made by distracted lab personnel, possible contamination of sterile areas, and in rare instances, there may be theft of supplies or intellectual property. But Dr. Anna Morales took her concerns to an extreme and always kept the laboratory door, cabinets and drawers locked, had all of her staff sign a confidentiality agreement, and made the IACUC contact her ahead of time when semi-annual inspections were due. This was not a problem for the IACUC because it routinely contacted investigators before performing its semiannual inspections.
Morales's protocol allowed her to bring mice up to her lab to perform a surgical procedure that required the use of the stereotaxic and recording equipment that was kept there. One of the staff working for Morales casually mentioned to an animal care technician that they were having some unexpected deaths of mice and the technician dutifully forwarded that information to the facility veterinarian. When the vet knocked on the door of Morales's lab she was told that she could not enter without Dr. Morales's permission and that Dr. Morales was gone for two more days. Not wanting to start an argument the veterinarian reported this to the IACUC, explaining that the attending veterinarian must have access to all animals.
When Morales returned there was a discussion between her, the veterinarian, and the IACUC chairman. Morales said that the veterinarian was not being denied access; rather, she was welcome to observe the surgery and the animals but unless there was a medical emergency she had to make an appointment to enter the lab. The veterinarian was adamant that she needed unfettered access to all animal care and use areas. The chairman didn't know what to say.

What is your opinion? Was Morales giving the veterinarian the access she said she needed or was the veterinarian right in demanding access whenever she believed it was needed?

\section{RESPONSE}

\section{Choose your battles}

\section{Madeline L. Budda, DVM, MS, DACLAM \& Michelle W. Staruch, BSc, CMAR, RLATG, RVT}

The veterinarian should have unrestricted access to all animals within the program. However, exerting this authority by force may not be the best approach. According to the Guide, "the institution must provide the [attending veterinarian] with sufficient authority, including access to all animals, and resources to manage the program of veterinary care" . If the investigator is not available during a pressing health problem, "the veterinarian must have the authority... to treat the animal, remove it from the experiment, institute appropriate measures to relieve severe pain or distress, or perform euthanasia"1. Unexpected deaths in Morales's lab merit prompt evaluation to determine if animals experienced unnecessary pain or distress.
But the lab has come to expect scheduled visits from the IACUC and they may not be aware of the veterinarian's authority to enter the lab unannounced.

In her effort to avoid an argument after being denied access to the lab, the veterinarian may have failed to fully communicate her intentions to evaluate animal care and possibly prevent unexpected animal deaths. Nonetheless, the veterinarian could still perform a preliminary evaluation without entering the lab. She could review copies of the animal records and perform necropsies of the deceased animals, then report her findings to the IACUC for further investigation. If the veterinarian suspected animal wellbeing was at risk based upon her preliminary findings, she could prevent more animals from leaving the facility until appropriate animal care practices could be confirmed in the lab. On the other hand, if her preliminary findings suggested good animal welfare was being upheld, she might feel more comfortable waiting a day or two to enter the lab by appointment.
Morales may feel warranted in limiting access to her lab. However, she should consider creating an exception for the veterinarian. Working with the veterinarian to promptly address animal care and use issues could help identify areas for improvement within the lab. Such collaboration could increase efficiency and help Morales's research stand up to peer review. The veterinarian should ask for a meeting with Morales to discuss their common goals and to educate the lab on best practices. An offer to sign one of Morales's confidentiality statements could help alleviate concerns about sensitive research information leaving the lab.

The IACUC chairman's response to this issue suggests that he may be unaware of the veterinarian's obligation to ensure animal care and wellbeing throughout the institution's animal program. According to PHS Policy, each institution must have at least one veterinarian "who has direct or delegated program authority and responsibility for activities involving animals at the institution" 2 . This could be remedied if the 